\section{Use of an in vitro model of hepatic steatosis for studying the anti-oxidant and anti- steatotic effects of fucoidan polysaccharides}

\section{Zeinab EI Rashed, ${ }^{1}$ Hala Khalife, ${ }^{2}$ \\ Adriana Voci, ${ }^{1}$ Elena Grasselli, ${ }^{1}$ \\ Laura Canesi, ${ }^{1}$ Ilaria Demori ${ }^{1}$}

1 Department of Earth, Environmental and Life Sciences, University of Genoa, Genoa, Italy; ${ }^{2}$ Rammal Rammal Laboratory (ATAC group), Faculty of Sciences l, Lebanese University, Lebanon

\section{Abstract}

Non Alcoholic Fatty Liver Disease (NAFLD) is characterised by fat accumulation in hepatocytes in the form of triacyglycerols (TAGs) within cytosolic lipid droplets. Fucoidans (FUs) are biologically active polysaccharides usually isolated from brown marine algae, but recently identified also in terrestrial plants. In this study, we aimed to investigate the anti-oxidant and anti-steatotic effects of FUs purified from C. compressa, F. hermonis, and E. globulus. To this aim, we used a validated NAFLD in vitro model consisting of rat hepatoma $\mathrm{FaO}$ cells exposed to an oleate/palmitate mixture. Such a model is suitable for rapid investigation of direct effects of natural and artificial compounds, together with satisfying the strategy of 3 Rs for laboratory use of animals. Our results indicated that all FUs display anti-oxidant and anti-steatotic activities. Steatotic $\mathrm{FaO}$ cells may be employed to further study the biological effects of FUs.

\section{Introduction}

Non Alcoholic Fatty Liver Disease (NAFLD) is the most common cause of liver disorders with high negative impact on human health. It is characterized by fat accumulation in more than $5 \%$ of hepatocyte, stored in the form of triacylglycerols (TAGs) within cytosolic lipid droplets (LDs), as a result of hepatic lipid metabolism unbalance. Molecular mechanisms and therapeutic strategies for NAFLD can be studied using in vivo models, which offer
Correspondence: Ilaria Demori, Department of Earth, Environmental and Life Sciences, University of Genoa, Genoa, Italy.

E-mail: idemori@unige.it

Key words: Non alcoholic fatty liver disease fucoidan; anti-steatotic; anti-oxidant.

Conference presentation: this paper was presented at the Second Centro 3R Annual Meeting - 3Rs in Italian Universities, 2019 , June 20-21, University of Genoa, Italy.

Received for publication: 28 October 2019. Accepted for publication: 11 November 2019

This work is licensed under a Creative Commons Attribution NonCommercial 4.0 License (CC BY-NC 4.0).

(C) Copyright: the Author(s), 2019

Licensee PAGEPress, Italy

Biomedical Science and Engineering 2019; 3(s3):109 doi:10.4081/bse.2019.109

the advantage to study the effects of active compounds at the level of the whole organism. However, major disadvantages are ethical concerns, requirement of skilled manpower, time consuming protocols and high costs, thus supporting the application of in vitro models which may satisfy the strategy
In vivo NAFLD model: High Fat Diet (HFD)-fed rats

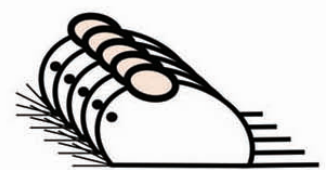

5 animals/group

1 wk acclimatization

5 wks of HFD to achieve steatosis
In vitro NAFLD model: rat hepatocyte primary cultures

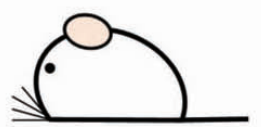

1 animal

$24 \mathrm{hrs}$ for cell seeding and attachment $24 \mathrm{hrs}$ of OP treatment to achieve steatosis
In vitro NAFLD model:

$\mathrm{FaO}$ rat hepatoma cell line

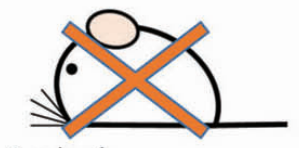

0 animal

$3 \mathrm{hrs}$ of OP treatment to achieve steatosis

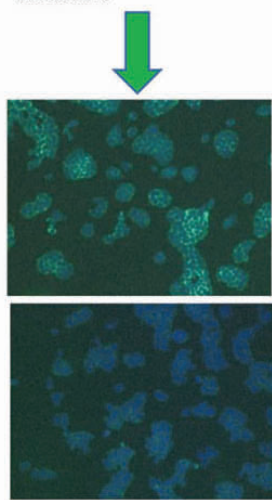

(A)

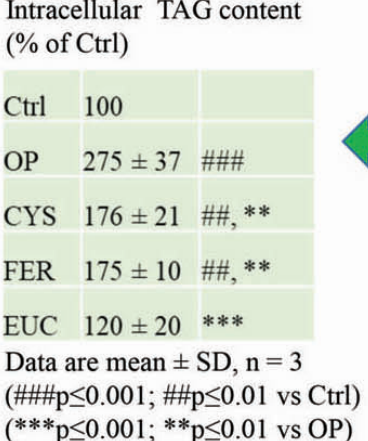

24 hrs of incubation with $50 \mu \mathrm{g} / \mathrm{mL}$ FUs
Bodipy/DAPI staining for LDs detection in OP cells (A) with respect to $\mathrm{Ctrl}(\mathrm{B})$

Figure 1. Schematic description of different in vivo and in vitro NAFLD experimental models with reference to the $3 \mathrm{R}$ strategy; the anti-steatotic effects of FUs extracted from CYS, FER and EUC is also shown. 
of 3 Rs (i.e. reduction, refinement and replacement) for laboratory use of animals. ${ }^{1}$ In the last years our group used both in vivo and in vitro models to study NAFLD, and demonstrated that the main markers of hepatic steatosis are maintained in all the experimental models, thus making the in vitro model suitable for rapid investigation of direct anti-steatotic effects of natural and artificial compounds. In the present study, we used an in vitro model of NAFLD to study the anti-oxidant and anti-steatotic effects of fucoidans (FUs). FUs are fucose rich polysaccharides predominantly found in the cell wall of brown algae such as Cystoseira compressa, but recently identified also in terrestrial plants such as Eucalyptus globulus and Ferula hermonis, growing in Lebanon.

\section{Materials and Methods}

FUs were purified from $C$. compressa (CYS), F. hermonis (FER), and E. globulus (EUC) as previously described. ${ }^{2}$ Anti-oxidant activity was determined by DPPH test. Steatotic rat hepatoma $\mathrm{FaO}$ cells were obtained by $3 \mathrm{~h}$ exposure to an oleate/palmitate (OP) mixture and then exposed to $50 \mu \mathrm{g} / \mathrm{mL}$ FUs for 24 h. ${ }^{1,3}$ The anti-steatotic effect of FUs was investigated by measuring intracellular TAG content and by detecting LD formation through fluorescence microscopy. The expression of PPAR (Peroxisome Proliferator Activated Receptor) isoforms and LD associated perilipins (PLINs) that play important roles in lipid homeostasis, was evaluated by qPCR. ${ }^{3}$

\section{Results}

The upper part of Figure 1 shows that a dramatic decrease in animal use and a high speeding up of the experimental procedures can be appreciated when comparing the number of animals needed and the duration of the treatments used in vivo and in vitro to obtain three different models of NAFLD. The lower part of Figure 1 shows the detection of LDs by Bodipy fluorescent staining in OP-treated $\mathrm{FaO}$ cells with respect to control (Ctrl) cells; the table shows that intracellular TAG content was significantly reduced in steatotic cells upon any FU treatment. This result was in line with a suppression of lipogenic genes such as PPAR $\gamma$, PLIN2 and PLIN5, which was measured by qPCR. DPPH assay allowed to demonstrate a significant anti-oxidant activity, which was exerted by FUs purified from all three species (IC 50 was $158.5 \pm 1.81,152.9 \pm$ $3.30,4.466 \pm 3.26 \mu \mathrm{g} / \mathrm{mL}$ for CYS, FER and EUC, respectively).

Of note, FUs purified from terrestrial plants exerted stronger anti-oxidant and anti-steatotic effects than those obtained from marine brown algae, with E. globulus FUs showing the highest activities.

\section{Conclusions}

Different in vivo and in vitro models have been established to resemble the most important features of NAFLD. In vitro approaches have the advantage of providing a simple and highly reproducible model, where the mechanisms can be studied directly at the cellular level. Our results validate the use of cell cultures as effectual tools to replace the use of laboratory animals when studying NAFLD and the effects and mechanisms of action of natural compounds with therapeutic potential, such as FUs. Steatotic FaO cells may be employed to further study the biological effects of FUs, particularly those extracted from $E$. globulus, which is confirmed as a powerful medicinal plant.

\section{References}

1. Grasselli E, Canesi L, Portincasa P, et al. Models of non-Alcoholic Fatty Liver Disease and Potential Translational Value: the Effects of 3,5-L-diiodothyronine. Ann Hepatol 2017;16:707-19.

2. Zein S, Haddad M, Krivoruchko E, et al. A New Molecule of Water-Soluble Polysaccharide Isolated from Eucalyptus Growing in Lebanon. Eur J Pharm Res 2018;1:48-54.

3. Grasselli E, Cortese K, Fabbri R, et al. Thyromimetic actions of tetrabromobisphenol A (TBBPA) in steatotic $\mathrm{FaO}$ rat hepatoma cells. Chemosphere 2014;112:511-8 